\title{
ON TWO-DIMENSIONAL QUASICONFORMAL GROUPS
}

\author{
PEKKA TUKIA
}

A quasiconformal group is a group $G$ of homeomorphisms of some open set $U$ in the $n$-ball $S^{n}$ such that each element of $G$ is $K$-quasiconformal for some fixed $K \geqq 1$. If we wish to specify $K$ and $U$, we say that $G$ is a $K$-quasiconformal group acting in $U$ (or of $U$ ).

One can obtain quasiconformal groups as follows. Let $V \subset S^{n}$ be open and let $G$ be a group of conformal homeomorphisms of $V$. (If $n \geqq 3$ and $V$ is connected, $G$ is a group of Möbius transformations.) Let $f: V \rightarrow U$ be quasiconformal. Then $f G f^{-1}$ is a quasiconformal group. It has been proposed by F. Gehring that all quasiconformal groups are of this form. We offer here a proof of this conjecture for quasiconformal groups acting in open subsets of the Riemann sphere.

Kuusalo [3, Theorem 3 p. 21] has proved the following theorem, which is related to ours. Let $S$ be a quasiconformal 2-manifold. Then $S$ has a conformal structure which is compatible with the quasiconformal structure of $S$. Quasiconformal groups have been considered also in Gehring-Palka [2]. The theory of quasiconformal mappings we need can be found in Lehto-Virtanen [4].

I wish to thank Kari Hag who read the manuscript and made many valuable remarks. In particular, she informed me of Maskit's work [5, 6], and the Corollary was suggested by her.

Theorem. Let $G$ be a $K$-quasiconformal group acting in an open subset $U \subset S^{2}$. Then there is a K-quasiconformal homeomorphism $h: U \rightarrow V \subset S^{2}$ such that $h G h^{-1}$ is a group of conformal self-maps of $V$ and that $h$ is the restriction of a K-quasiconformal homeomorphism $f$ of $S^{2}$ whose complex dilatation $\mu_{f}$ vanishes a.e. outside $U$.

A consequence of the above theorem is that if the fundamental group of a plane domain $U$ is non-cyclic and $G$ is a quasiconformal group of $U, G$ is discrete, i.e. there are no sequences $f_{i} \in G \backslash\{\mathrm{id}\}, i \geqq 0$, such that $\lim _{i \rightarrow \infty} f_{i}=\mathrm{id}$. This follows since the group of conformal self-maps of a plane domain with non-cyclic fundamental group is discrete. This is in turn a consequence of the theory of Fuchsian groups, since in this case the universal cover of $U$ is the open unit disk and the limit set of the cover translation group contains more than two points.

If $V \subset S^{2}$ is open and connected, there is a conformal homeomorphism $g: V \rightarrow V^{\prime} \subset S^{2}$ such that $g \alpha g^{-1}$ is a Möbius transformation of $V^{\prime}$ whenever $\alpha$ is 
a conformal self-map of $V$, cf. Maskit [5,6]; if $V$ is simply connected, this follows by Riemann's mapping theorem. Therefore we have the following

Corollary. Let $G$ and $U$ be as in the above theorem and assume that $U$ is connected. Then there is a K-quasiconformal homeomorphism $h^{\prime}: U \rightarrow U^{\prime} \subset S^{2}$ such that $h^{\prime} G h^{-1}$ is a group of Möbius transformations of $U^{\prime}$.

Note that in general we cannot extend $h^{\prime}$ to a quasiconformal homeomorphism of $S^{2}$.

Proof of the theorem. We find the conditions for $\mu$ guaranteeing that if a quasiconformal map $f$ of $S^{2}$ has the complex dilatation $\mu$, then $f$ is a solution of our problem. Clearly, we must set

$$
\mu \mid S^{2 \backslash} U=0 .
$$

The conformality of $f \circ g \circ\left(f^{-1} \mid f(U)\right)$ for $g \in G$ is equivalent to the validity of

$$
\mu_{f}(x)=\mu_{f g}(x)
$$

for almost all $x \in U$ when $g \in G$ is fixed. Computing $\mu_{f g}$ in terms of $\mu_{f}$ and $\mu_{g}$ we must have

$$
\mu_{f}(x)=\frac{\mu_{g}(x)+\mu_{f}(g(x)) e^{-2 i \arg g_{z}(x)}}{1+\mu_{g}(x) \mu_{f}(g(x)) e^{-2 i \arg g_{\bar{z}}(x)}}
$$

a.e. in $U$. If we can find a measurable function $\mu: S^{2} \rightarrow C$ with $\|\mu\|_{\infty} \leqq(K-1) /(K+1)$ that satisfies (1) and (3) for $\mu=\mu_{f}$ a.e. in $U$ if $g \in G$ is given, then any homeomorphic solution $f$ of the equation

$$
\mu_{f}=\mu \quad \text { a.e. in } S^{2}
$$

is also a solution of our problem.

We write (3) in the form

$$
\mu_{f}(x)=T_{g}(x)\left(\mu_{f}(g(x))\right)=\mu_{f g}(x),
$$

where

$$
T_{g}(x)(z)=\frac{\mu_{g}(x)+e^{-2 i \arg g_{z}(x)} z}{1+\mu_{g}(x) e^{-2 i \arg g_{\bar{z}}(x)} z}=\frac{a+\bar{b} z}{b+\bar{a} z}
$$

with $a=g_{\bar{z}}(x), b=g_{z}(x)$. Thus, whenever defined (i.e. a.e. in $U$ for fixed $g$ ), $T_{g}(x)$ is a conformal self-map of the open unit disk $D$ and is an isometry in the hyperbolic metric.

Consider the sets $M_{x}=\left\{\mu_{g}(x): g \in G\right\}, x \in U$. By (5) we have

$$
\begin{aligned}
T_{g}(x)\left(M_{g(x)}\right) & =\left\{T_{g}(x)\left(\mu_{g^{\prime}}(g(x))\right): g^{\prime} \in G\right\} \\
& =\left\{\mu_{g^{\prime} g}(x): g^{\prime} \in G\right\}=\left\{\mu_{g^{\prime}}(x): g^{\prime} \in G\right\} \\
& =M_{x}
\end{aligned}
$$

for almost all $x \in U$ and every $g \in G$ if $G$ is countable. Let us assume that there is a map $X \mapsto P(X) \in D$ that assigns a point to every non-empty subset $X \subset D$ which is bounded in the hyperbolic metric in such a way that $P(g(X))=g(P(X))$ for 
every isometry $g$ of $D$. Then, for countable $G$, the map $\mu(x)=P\left(M_{x}\right)$ satisfies (3) (with $\mu_{f}=\mu$ ) a.e. in $U$ for all $g \in G$.

Now we construct such a map $P$. Let $X \subset D$ be bounded, $X \neq \emptyset$. Then there is a unique closed hyperbolic disk $D(x, r)$ with center $x$ and radius $r \geqq 0$ with the properties

(i) $D(x, r) \supset X$, and

(ii) if $D\left(y, r^{\prime}\right) \supset X, y \neq x$, then $r^{\prime}>r$.

To see the existence of $D(x, r)$ we can reason as follows. In any case there is a smallest $r \geqq 0$ such that if $r^{\prime}>r$, there is $y \in D$ with $D\left(y, r^{\prime}\right) \supset X$. Next it is easy to see that there is at least one $x \in D$ such that $D(x, r) \supset X$. Assume that there is another point $y \in D$ with $D(y, r) \supset X$. Let $w$ be one of the two points of $\partial D(x, r) \cap \partial D(y, r)$ and let $z$ be the orthogonal projection (in hyperbolic geometry) of $w$ onto the hyperbolic line through $x$ and $y$. Consider the hyperbolic triangle with vertices $x, z$ and $w$. It has a right angle at $z$ and therefore it is geometrically evident that $d(x, w)=$ $r>d(z, w)$. This follows also from the relation $\cosh r=\cosh d(x, z) \cosh d(z, w)$ (cf. e.g. Coxeter [1]). But then, if $r^{\prime}=d(z, w), r^{\prime}<r$ and $D\left(z, r^{\prime}\right) \supset D(x, r) \cap$ $D(y, r) \supset X$. This proves the uniqueness of $x$. Therefore, if we let $P(X)$ be the center of the smallest closed hyperbolic disk containing $X$, we have a well-defined map $P$. Clearly, $P(g(X))=g(P(X))$ for any isometry $g$ of $D$. It has also the following property.

(A) If $X \subset D(y, s), X \neq \emptyset$, then $P(X) \in D(y, s)$.

To see the validity of (A), note first that $r \leqq s$ if $r$ is the radius of the smallest disk containing $X$. Then, if $d(y, P(X))>s$, we can reason as above and find $D\left(z, r^{\prime}\right) \supset X$ with $r^{\prime}<r$. Therefore $d(y, P(X)) \leqq s$.

Now we assume for a moment that $G$ is countable, $G=\left\{g_{0}, g_{1}, \ldots\right\}$. We define a map $\mu$ by setting

$$
\begin{gathered}
\mu \mid S^{2} \backslash U=0, \quad \text { and } \\
\mu(x)=P\left(M_{x}\right) \quad \text { if } \quad x \in U,
\end{gathered}
$$

which defines $\mu$ a.e. in $S^{2}$. Since $M_{x} \subset D(0, r)$, where $r=d(0,(K-1) /(K+1))$, for almost all $x \in U,\|\mu\|_{\infty} \leqq(K-1) /(K+1)$ by (A). We have already observed that $\mu$ satisfies (3) (with $\mu_{f}=\mu$ ) a.e. in $U$ for all $g \in G$. It is also measurable. To see this, let

$$
\mu_{n}(x)=P\left(\left\{\mu_{g_{i}}(x): i \leqq n\right\}\right)
$$

if $x \in U$ and $n \geqq 0$. Then $\mu_{n}$ is a.e. defined and it is certainly measurable. Since $\mu(x)=\lim _{n \rightarrow \infty} \mu_{n}(x)$ a.e. in $U$, also $\mu$ is measurable. Therefore, if $G$ is countable, there is a map $f$ satisfying the conditions of the theorem.

If $G$ is not countable, there is always a countable subgroup $G^{\prime} \subset G$ which is dense in the topology of uniform convergence in compact sets. This follows from the separability of the set of all continuous maps $U \rightarrow S^{2}$ in this topology (they can be approximated by PL maps). Then if $f$ satisfies the conditions of the theorem with respect to $G^{\prime}$, it satisfies them also with respect to $G$. 
Remarks. An earlier version of this note proved the preceding theorem under the assumptions that $G$ was discrete and that the limit set of $G$ had zero measure. Unable to find a solution of (3) with $\mu=\mu_{f}$ for general $G$, I had to make these assumptions on which one can find a measurable fundamental set $S$ for $G$, and set $\mu \mid S=0$, determining $\mu$ completely. After it was written, F. W. Gehring called my attention to the paper [7] by Sullivan. This paper contains a sketch of the proof of the above theorem. Sullivan's proof differs from ours in the definition of $\mu$; we have defined $\mu(x)=P\left(M_{x}\right)$ whereas Sullivan sets $\mu(x)=B\left(M_{x}\right)$, where $B(X)$ is the barycenter of the convex hull of $X$, both in hyperbolic geometry. Since Sullivan gives only the barest outline of the proof and since he makes also some unnecessary assumptions (e.g. $G$ was assumed to be discrete), the publication of this little note is perhaps justified.

The use of the map $P$ seems also to have some slight advantages over the use of the map $B$. No doubt one can take barycenters also in hyperbolic geometry, but to prove the existence of $B(X)$ is non-trivial, whereas this proof is very simple for $P(X)$. Secondly, $X \mapsto P(X)$ is continuous but $X \mapsto B(X)$ is not. That is, if $\varepsilon>0$ is given, there is $\delta=\delta(\varepsilon)>0$ such that if $X, Y \subset D$ are non-empty and bounded and if

$$
\varrho(X, Y)=\sup \{d(x, Y), d(X, y): x \in X, y \in Y\}<\delta,
$$

then $d(P(X), P(Y))<\varepsilon$; cf. (8) below. To see the discontinuity of $B$, let $A=\{0,1\}$ and $A_{n}=\{0,1,1+i / n\} \subset C, n>0$. Then if we take the barycenter of the convex hull in the euclidean geometry of $C$, we have $B(A)=1 / 2$ but $\lim _{n \rightarrow \infty} B\left(A_{n}\right)=2 / 3$.

Appendix 1. It is easy to derive an estimate for $d(P(X), P(Y))$ in terms of $\varrho(X, Y)$, and since we will need it in a future paper, we do it here. A consequence of this estimate is that if the family $\left\{\mu_{g}: g \in G\right\}$ is equicontinuous, $\mu_{f} \mid U$ is continuous. Let $X, Y \subset D$ be non-empty and bounded. Let $d=\varrho(X, Y), x=P(X)$, $y=P(Y)$, and let $D(x, r) \supset X$ and $D\left(y, r^{\prime}\right) \supset Y$ be the smallest disks containing $X$ and $Y$, respectively. Then $D(x, r+d) \supset Y$, implying $r^{\prime} \leqq r+d$. Similarly, $D\left(y, r^{\prime}+d\right) \supset X$, and therefore $D(y, r+2 d) \supset X$. We consider the disks $D(x, r) \supset X$ and $D(y, r+2 d) \supset X$. If $2 d \leqq d(x, y) \leqq 2 r+2 d, \partial D(x, r) \cap \partial D(y, r+2 d) \neq \emptyset$. We assume now that $d(x, y)>2 d$. Since $D(x, r+d) \supset Y$, by (A) $y \in D(x, r+d)$, implying $d(x, y) \leqq r+d$. Therefore there is a point $w \in \partial D(x, r) \cap \partial D(y, r+2 d)$. Consider the hyperbolic triangle $T$ with vertices $x, y$ and $w$. Let $z$ be the orthogonal projection (in hyperbolic geometry) of $w$ onto the hyperbolic line through $x$ and $y$. If $z \in T, z \neq x$, then $r^{\prime \prime}=d(z, w)<r$, and $D\left(z, r^{\prime \prime}\right) \supset D(x, r) \cap D(y, r+2 d) \supset X$, contradicting the definition of $r$. Therefore $z \notin T \backslash\{x\}$, i.e., $\varphi \geqq \pi / 2$ when $\varphi$ is the angle of $T$ at $x$. Now, keep $r$ and $r+2 d$ fixed and decrease $\varphi$ from $\pi$ to $\pi / 2$. Then $d(x, y)$ increases from $2 d$ to a value $d^{\prime}$ with $\cosh (r+2 d)=\cosh r \cosh d^{\prime}$. This is geometrically evident and follows also from the relation $\cosh (r+2 d)=\cosh r \cosh d(x, y)-$ $\sinh r \sinh d(x, y) \cos \varphi ;$ cf. [1]. It follows

$$
\cosh d(x, y) \leqq \cosh (r+2 d) / \cosh r .
$$


This is also valid if $d(x, y) \leqq 2 d$, which case we have excluded from the above discussion.

We have $\cosh (r+2 d) / \cosh r=e^{2 d}\left(1+e^{-2(r+2 d)}\right) /\left(1+e^{-2 r}\right) \leqq e^{2 d}$. Thus, substituting back into (6) $d=\varrho(X, Y), x=P(X)$ and $y=P(Y)$, we get

$$
\begin{aligned}
d(P(X), P(Y)) & \leqq \operatorname{arcosh} e^{2 \varrho(X, Y)}=\log \left(e^{2 \varrho(X, Y)}+\left(e^{4 \varrho(X, Y)}-1\right)^{1 / 2}\right) \\
& <2 \varrho(X, Y)+\log 2 .
\end{aligned}
$$

If $\varrho(X, Y)$ is small, we get a Hölder-type inequality. Let $c=e^{2 \varrho(X, Y)}-1$. Then $\log \left(e^{2 \varrho(X, Y)}+\left(e^{4 \varrho(X, Y)}-1\right)^{1 / 2}\right)=\log \left(1+c+\left(2 c+c^{2}\right)^{1 / 2}\right)=\log \left(1+c^{1 / 2}\left(c^{1 / 2}+(2+c)^{1 / 2}\right)\right) \leqq$ $c^{1 / 2}\left(c^{1 / 2}+(2+c)^{1 / 2}\right)$. If $\varrho(X, Y) \leqq R, c \leqq 2 e^{2 R} \varrho(X, Y)$. We have then by $(7)$

$$
d(P(X), P(Y)) \leqq C(R) \varrho(X, Y)^{1 / 2} \quad \text { if } \varrho(X, Y) \leqq R,
$$

where $C(R)=2 e^{R}\left(\left(R e^{2 R}\right)^{1 / 2}+\left(1+R e^{2 R}\right)^{1 / 2}\right)$.

Note that $P(X)$ exists and that (6), (7), (8) and (A) are valid also if $X$ and $Y$ are non-empty bounded subsets of the $n$-dimensional hyperbolic space.

Appendix 2. (Added December 1979.) It is possible to give a sharper estimate for the dilatation of the map $f$ of the preceding Theorem. In fact,

$f$ is $K^{\prime}$-quasiconformal where $K^{\prime}=(\sqrt{K+1 / K}+\sqrt{K}-1 / \sqrt{K}) / \sqrt{2} \leqq \min \left(K^{1 / \sqrt{2}}, \sqrt{2 K}\right)$.

This is an immediate consequence of the following lemma. Note that always $0=\mu_{\text {id }}(z) \in M_{z}$ and that $d(0,(K-1) /(K+1))=\log K$ when the hyperbolic metric of $D$ is given by $2|d z| /\left(1-|z|^{2}\right)$ in which the formulae of hyperbolic trigonometry are valid.

Lemma. Let $X \subset D(0, r), r \geqq 0$, and assume that $0 \in X$. Then the center of the smallest hyperbolic disk containing $X$ satisfies

$$
d(0, P(X)) \leqq \beta(r)=\operatorname{arcosh}(\cosh r)^{1 / 2} .
$$

We have the following relations for the function $\beta: r / 2<\beta(r)<r / \sqrt{2}$ if $r>0$, $\beta(r)<r / 2+\log \sqrt{2}$ and $\lim _{r \rightarrow \infty}(\beta(r)-r / 2)=\log \sqrt{2}$.

Proof. Let $D(x, \varrho)$ be the smallest hyperbolic disk containing $X$. We can assume that $x \in R, x \geqq 0$. It also suffices to consider the case $d(0, x)>r / 2$; by (A) always $x \in D(0, r)$. Then

$$
r / 2<d(0, x) \leqq \varrho \leqq r,
$$

since $0 \in D(x, \varrho)$ and in any case $\varrho \leqq r$. Thus $\partial D(x, \varrho) \cap \partial D(0, r)$ consists of two points; let $z$ be one of them. Let $w$ be the orthogonal projection (in hyperbolic geometry) of $z$ onto $R \cap D$ (=the hyperbolic line joining 0 and $x$ ). We consider the following three cases
( $\alpha) \quad w \leqq 0 ;$
(B) $0<w<x$;
( $\gamma) \quad w \geqq x$.

Let $T$ be the hyperbolic triangle with vertices $0, x$ and $z$. In case $(\alpha)$ the angle of $T$ at $0 \geqq \pi / 2$. Therefore [1, eq. 12.94] $\cosh \varrho=\cosh d(x, z) \geqq \cosh d(0, x) \cosh d(0, z)=$ $\cosh d(0, x) \cosh r$. This implies $\varrho>r$ which is impossible by (9). Thus $(\alpha)$ is impos- 
sible. Case $(\beta)$ cannot occur either, since now $D(w, d(w, z)) \supset D(0, r) \cap D(x, \varrho) \supset X$. This is impossible since the triangle with vertices $x, z$ and $w$ has a right angle at $w$ and thus $d(w, z)<d(x, z)=\varrho$.

Thus, if $d(0, x)>r / 2,(\gamma)$ is the only possibility. Now the angle of $T$ at $x \geqq \pi / 2$. This, together with (9), implies $\cosh r=\cosh d(0, z) \geqq \cosh d(0, x) \cosh d(x, z)=$ $\cosh d(0, x) \cosh \varrho \geqq \cosh ^{2} d(0, x)$. Thus $d(0, x) \leqq$ ar $\cosh (\cosh r)^{1 / 2}$, proving the inequality for $d(0, P(X))=d(0, x)$.

We then examine the properties of $\beta(r)$. Differentiating $\beta(r)$, we get

$$
\beta^{\prime}(r)=\frac{\cosh r / 2}{\sqrt{2 \cosh r}}=\sqrt{\frac{e^{r}+e^{-r}+2}{4\left(e^{r}+e^{-r}\right)}} \in(1 / 2,1 / \sqrt{2})
$$

if $r>0$, proving the first inequalities for $\beta$. We get the next, since

$$
\begin{aligned}
\beta(r) & =\log \left(\sqrt{\left(e^{r}+e^{-r}\right) / 2}+\sqrt{\left(e^{r}+e^{-r}\right) / 2-1}\right) \\
& =\log \left(\sqrt{e^{r}+e^{-r}}+e^{r / 2}-e^{-r / 2}\right)-\log \sqrt{2} \\
& <\log \left(e^{r / 2}+e^{-r / 2}+e^{r / 2}-e^{-r / 2}\right)-\log \sqrt{2}=r / 2+\log \sqrt{2} .
\end{aligned}
$$

Finally, the above expression for $\beta(r)$ gives immediately $\lim _{r \rightarrow \infty}(\beta(r)-r / 2)=\log \sqrt{2}$.

We remark that the function $\beta$ is best possible in the above lemma. In fact, let $T$ be the triangle with vertices $0, x$ and $y$ where $x, y \in \partial D(0, r)$ and $T$ has equal angles at $x$ and $y$. Choose these angles in such a way that if $t$ is the orthogonal projection of 0 onto the opposite side, we have $d(t, 0)=d(t, x)=d(t, y)$. Then $d(0, t)=$ $d(0, P(T))=\beta(r)$.

\section{References}

[1] Coxeter, H. S. M.: Non-Euclidean geometry. - The University of Toronto Press, Toronto, Canada, 1947.

[2] Gehring, F. W., and B. Palka: Quasiconformally homogeneous domains. - J. Analyse Math. 30, 1976, 172-199.

[3] Kuusalo, T.: Verallgemeinerter Riemannscher Abbildungssatz und quasikonforme Mannigfaltigkeiten. - Ann. Acad. Sci. Fenn. Ser. A I 409, 1967, 1-24.

[4] Lehto, O., and K. I. Virtanen: Quasiconformal mappings in the plane. - Springer-Verlag, Berlin-Heidelberg-New York, 1973.

[5] Maskit, B.: Construction of Kleinian groups. - Proceedings of the Conference on Complex Analysis, Minneapolis 1964, edited by A. Aeppli, E. Calabi, and H. Röhrl, SpringerVerlag, Berlin-Heidelberg-New York, 1965, 281-296.

[6] Maskit, B.: The conformal group of a plane domain. - Amer. J. Math. 90, 1968, 718-722.

[7] Sullivan, D.: On the ergodic theory at infinity of an arbitrary discrete group of hyperbolic motions. - Proceedings of the Stony Brook Conference on Riemann surfaces and Kleinian groups, June 1978 (to appear).

University of Helsinki

Department of Mathematics

SF-00100 Helsinki 10

Finland

Received 18 April 1979

Revision received 15 August 1979 